\title{
EFECTO DE LA FERMENTACIÓN DE CACAO (Theobroma cacao L.), VARIEDAD NACIONAL Y TRINITARIO, EN CAJAS DE MADERAS NO CONVENCIONALES SOBRE LA CALIDAD FÍSICA Y SENSORIAL DEL LICOR DE CACAO
}

EFFECT OF THE FERMENTATION OF CACAO (Theobroma cacao L.), NATIONAL AND TRINITARY VARIETY, IN NON-CONVENTIONAL WOOD BOXES ON THE PHYSICAL AND SENSORY QUALITY OF CACAO LIQUOR

\section{Cyntia Erazo Solórzano; Kerly Bravo Franco; Diego Tuárez García; Ángel Fernández Escobar; Yenny Torres Navarrete; Jaime Vera Chang.}

Universidad Técnica Estatal de Quevedo, Facultad de Ciencias Pecuarias, Av. Walter Andrade, km 1,5 vía a Santo Domingo, C.P. 73. Quevedo-Ecuador

Email:cerazo@uteq.edu.ec

https://doi.org/10.33789/talentos.8.2.153

Resumen: La investigación se llevó a cabo en la Universidad Técnica Estatal de Quevedo. Se valoró la influencia de fermentadores de cacao elaborados de maderas (Guayacán Blanco, Laurel, Pino) sobre la calidad físico-químicas de las almendras de cacao variedad Trinitario y Nacional; análisis sensorial y bromatológica al licor de cacao. Se aplicó un diseño completamente al azar (DCA) con arreglo factorial (3x2), se determinó el porcentaje de fermentación en las dos variedades visualizando el $60 \%$ mínimo de almendras bien fermentadas, granos violetas $21 \%$, granos pizarrosos superó el $12 \%$, en cuanto al perfil de sabores específicos logró determinarse que el T1 (C. Nacional-C. Laurel) olor a Cacao, T6 (C. Trinitario-Pino) perfil Floral, T2 (C. Trinitario-Guayacán Blanco) Dulce y presenta los mejores atributos; en cuanto al perfil de sabores básicos amargor el T3 (C. Nacional-Pino) tiene promedio alto; el T6 y T5 (NacionalGuayacán Blanco) obtiene una media en acidez y astringencia; en sabores Verde y Moho el T6 y T3 presentan promedios altos en el licor de cacao. Referente a los análisis físicos químicos, el T6 presenta humedad (2,44\%), el T5 (3,37\%) en ceniza, (51,11\%) en grasa el T4 (Trinitario- 
Laurel), el T6 con un pH de $(5,82)$ y T5 presenta una acidez de (1,34\%) en el licor de cacao. La temperatura máxima alcanzada en la fermentación del cacao Nacional $\left(44,52{ }^{\circ} \mathrm{C}\right)$ en cajones de madera Pino, mientras que la temperatura mínima fue en el cacao Trinitario $\left(35,66^{\circ} \mathrm{C}\right)$ en el fermentador elaborado de madera Guayacán Blanco.

Palabras claves: amargor, atributos, astringencia, prueba de corte, fermentador.

\begin{abstract}
The research was carried out at the Quevedo State Technical University. The influence of cocoa fermenters made from wood (Guayacán Blanco, Laurel, Pino) on the physicalchemical quality of the Trinitario and Nacional variety cocoa beans was assessed; sensory and bromatological analysis of cocoa liquor. A completely randomized design (DCA) was applied with a factorial arrangement (3x2), the minimum $60 \%$ of well-fermented almonds was determined by means of the cutting test, $21 \%$ violet grains, slate grains above $12 \%$, regarding the profile of Specific flavors were able to determine that T1 (C. Nacional-C. Laurel) smell of Cacao, T6 (C. Trinitario-Pino) Floral profile, T2 (C. Trinitario-Guayacán Blanco) Sweet and presents the best attributes; Regarding the profile of basic bitterness flavors, T3 (C. NacionalPino) has a high average; T6 and T5 (Nacional-Guayacán Blanco) obtain an average in acidity and astringency; In acquired Green and Mold flavors, T6 and T3 show high averages in cocoa liquor. Regarding the physical and chemical analyzes, T6 presents humidity (2.44\%), T5 (3.37 $\%)$ in ash, (51.11\%) in fat, T4 (Trinitario-Laurel), T6 with a pH of (5.82) and T5 presents an acidity of $(1.34 \%)$ in the cocoa liquor. The maximum temperature reached in the fermentation of the Nacional cocoa $\left(44.52^{\circ} \mathrm{C}\right)$ in pine wood crates, while the minimum temperature was in the Trinitario cocoa $\left(35.66^{\circ} \mathrm{C}\right)$ in the fermenter made of Guayacán Blanco wood.
\end{abstract}

Keywords: bitterness, attributes, astringency, shear test, fermenter.

\section{INTRODUCCIÓN}

Ecuador tiene la característica de producir cacao fino y de aroma con una oferta de 60 a 70 mil toneladas al año. Hace algún tiempo este producto representó el $80 \%$ de producción en todo el mundo. El $60 \%$ de producción de cacao fino y de aroma le corresponde a Ecuador lo cual es reconocido por otros países productores de cacao. Esto se debe a la presencia de cacao llamado "Nacional" originario del Forastero Amazónico (Ordoñez et al., 2019).
Las variedades de cacao, Nacional y Trinitario son las que predominan en las plantaciones cacaoteras del Ecuador, de las cuales, a la variedad de cacao Nacional se le ha reconocido mundialmente por sus características de sabor y aroma, apreciadas en la preparación de chocolates, contrastándose con la variedad Trinitario la cual no alcanza las características organolépticas del cacao Nacional (Morales et al., 2016).

La fermentación del cacao es una etapa muy importante en el procesamiento del grano, ya que se producen cambios bioquímicos 
que dan origen a los precursores del aroma y sabor, lo que determina su calidad física y química. Entre los cambios bioquímicos está el desarrollo de la pigmentación color marrón a partir de compuestos fenólicos, lo cual es un indicativo de la fermentación del grano de cacao. Además, los contenidos de precursores sensoriales como poli fenoles alcaloides (cafeína y teobromina) y acidez volátil (en especial el ácido acético), son indicadores de la calidad organoléptica del cacao (Rivera Fernández et al., 2012).

La calidad del cacao radica principalmente en la etapa de pos cosecha, tiempo en el cual las almendras de cacao son tratadas en condiciones adecuadas con el fin de cumplir una serie de transformaciones bioquímicas que favorecen la calidad organoléptica (aroma y sabor). La fermentación del cacao es una etapa indispensable, en la cual la pulpa que envuelve las semillas son metabolizadas por microorganismos que producen compuestos como el etanol y ácido acético los cuales promueven cambios fisicoquímicos importantes en las almendras (Ordoñez et al., 2019).

El comercio de productos primarios, entre ellos el cacao en grano, es de gran importancia para la economía del Ecuador y de la mayoría de países en desarrollo, se obtiene como subproductos a través de procesos industriales la pasta, licor, manteca, torta y el polvo de cacao, que son consumidos por la población. En la actualidad la demanda está orientada en materia prima orgánica libre de toxicidades y que posean características de calidad, por este motivo es significativo caracterizar los parámetros de perfiles sensoriales. La calidad comprende en seleccionar frutos sanos, ubicar en recipientes especiales y en condiciones apropiadas, sufren transformaciones físicoquímicas lo que permite desarrollar perfiles sensoriales propias del chocolate (Vallejo et al., 2018).

Según Palencia, "en los procesos de investigación se ha encontrado que la madera permite obtener un mejor sabor y una mejor coloración del grano. Se han hecho varios ensayos donde se trabajan cajones plásticos y de caucho, pero no se han obtenido los resultados deseados, conservar ese aroma y ese sabor de las almendras" (Giraldo Palencia, 2016).

Por lo expuesto, la presente investigación tiene como propósito evaluar el efecto de la micro fermentación de cacao (Theobroma cacao L.) variedad Nacional y Trinitario en maderas no convencionales sobre la calidad física y sensorial del licor de cacao con el fin de utilizar nuevos cajas fermentadoras para favorecer la calidad de las características organolépticas del producto final, se realizó los análisis fisicoquímicos y se evaluó el perfil sensorial del licor de cacao, todos los análisis se realizaron en el laboratorio Bromatológico de la Universidad Técnica Estatal de Quevedo (UTEQ) finca experimental "La María".

\section{MATERIALES Y MÉTODOS}

La presente investigación se realizó en la UTA, Facultad de Ciencias Pecuarias en la Finca Experimental "La María", está ubicada en el kilómetro 7 1 $1 / 2$ de la vía Quevedo- El Empalme, Provincia de Los Ríos, cuya situación geográfica es de $01^{\circ} 06^{\prime}$ de latitud 
Sur y $79^{\circ} 29^{\prime}$ de longitud Oeste a una altura de $75 \mathrm{msnm}$.

El cacao fue obtenido en el recinto Fayta, de la parroquia San Carlos perteneciente al cantón Quevedo de la provincia de Los Ríos, finca "La Represa", cuya ubicación geográfica es de $1^{\circ} 03^{\prime} 18^{\prime \prime}$ de latitud Sur y de $79^{\circ} 25^{\prime} 24^{\prime \prime}$ de longitud Oeste a una altura de $90 \mathrm{msnm}$, propiedad de la UTEQ. Se utilizó 2 kilogramos de cacao Nacional y Trinitario por cada tratamiento y se construyeron fermentadores de cacao con los diferentes tipos de maderas en estudio; la investigación fue de carácter exploratoria, descriptiva y experimental, puesto a que no se ha encontrado datos sobre la utilización de maderas no convencionales en la fermentación de cacao.

\subsection{Análisis estadístico}

Se empleó un diseño completamente al azar (DCA) con arreglo factorial (3 x 2), como factor A tres tipos de maderas no convencionales (Laurel, Pino, Guayacán blanco) para la fermentación y como factor B dos variedades de cacao Nacional y Trinitario, con 6 tratamientos y 4 repeticiones con un total de 24 unidades experimentales. Para la comparación de la media de los tratamientos se utilizó la prueba de rangos múltiples de Tukey $(\mathrm{p} \leq 0,05)$.

\subsection{Descripción del proceso}

\section{Recepción}

El criterio de recolección de las mazorcas de cacao, se basó en que estuvieran, sanas y en estado de madurez fisiológica, después se realizó dos cortes, a la mitad de la mazorca y dos cortes en la parte superior e inferior, de esta manera facilitó la obtención o extracción de las almendras de cacao.

\section{Peso del grano}

Luego de obtener los granos o almendras de cacao se procedió a pesar los $2 \mathrm{~kg}$ de cacao en baba de cada variedad, para posteriormente colocar en los fermentadores.

\section{Fermentación}

Se colocaron las almendras en los fermentadores, fueron cubiertas con hojas de plátano, yute y en la parte superior con plástico negro para evitar que ingrese algún tipo de insecto. Se registró la hora de inicio de la fermentación, a las 48 horas se realizó el primer volteo en las dos variedades en estudio y posterior cada 24 horas hasta que finalizó la etapa de fermentación (entre 5 y 7 días), los volteos fueron de forma manual, con ayuda de un palo o caña pequeña, cuidadosamente para no ocasionar daños en los granos, diariamente se controló la temperatura $\left(35-44^{\circ} \mathrm{C}\right)$ a cada tratamiento.

\section{Secado}

El método del secado se realizó de manera natural, es decir, los granos fueron expuesto al sol; los primeros días se secó al sol por 2 o 3 horas removiendo los granos, a partir del tercer día se incrementó las horas de secado de 5 a 6 horas removiendo cada hora por 5 días, para saber si el secado había finalizado, se tomaron muestras de las almendras para determinar el porcentaje de humedad en el grano (Instituto Ecuatoriano de Normalizacion INEN, 2013). En el secado es importante reducir hasta el 7 $\%$ para su correcto almacenamiento (Chang, 2018). 


\section{Tostado}

El proceso de tostado se realizó a una temperatura de $\left(120-135^{\circ} \mathrm{C}\right)$ por 25 minutos.

\section{Descascarillado}

Se realizó de forma manual retirando la cascarilla que recubre la almendra.

\section{Molienda}

Las almendras de cacao se ingresaron al molino MTC 250 para reducir el tamaño de partícula. En este proceso el cacao empezó a desprender su contenido de grasa el cual se fue transformando en pasta o licor de cacao.

\subsection{Análisis físico - químico}

\section{Humedad}

Contenido de humedad del licor de cacao, es la pérdida de la masa determinada por el método específico en la norma técnica expresado como un porcentaje en masa; se pesaron 10 $\mathrm{g}$ de muestra, se colocaron en un crisol la muestra triturada (evitando la formación de pasta), colocando en la estufa a $105^{\circ} \mathrm{C}$ por 16 horas, luego se colocó en el desecador hasta que se enfriará y pesar el resultante (Servicio Ecuatoriano de Normalización INEN, 2017).

\section{Porcentaje de fermentación}

Para valorar el porcentaje de fermentación de los granos se realizó la prueba de corte, para lo cual se recolecto al azar 100 almendras de cacao, se pesaron y se cortó de manera longitudinal el grano; y de acuerdo a las características físicas internas se determinó el porcentaje fermentación que presenta la almendra acorde a la norma INEN 176:2018 (Instituto Ecuatoriano de Normalización, 2018).

\section{Determinación de pH}

Para determinación del potencial de hidrogeno se realizó lo siguiente:

Se vertió 10 gramos de muestra en $90 \mathrm{ml}$ de agua destilada en un vaso de precipitación. Se mezcló la muestra con el agua destilada, se ubicó el potenciómetro en la muestra y se procedió a tomar la lectura (Association of Analytical Communities, 2020).

\section{Determinación de Acidez Titulable}

Se tomó 10 gramos de muestra, introduciéndola en un matraz volumétrico de $250 \mathrm{ml}$, se añadió $50 \mathrm{ml}$ de agua destilada, agitando vigorosamente. Se realizó la titulación con Hidróxido de sodio y como indicador fenolftaleína (Instituto Ecuatoriano de Normalización INEN, 2006)

\section{Determinación de Ceniza}

Se introdujo los crisoles durante $30 \mathrm{~min}$ en la estufa, con la finalidad de eliminar humedad existente en los crisoles, posteriormente se pesó 1 gr de muestra con aproximación al 0,1 mg, y se llevó a la mufla a $600{ }^{\circ} \mathrm{C}$ por tres horas. Transcurrido ese tiempo, se dejó enfriar en el desecador por media hora, pesar con precisión y realizar los cálculos respectivos (Instituto Ecuatoriano de Normalización, 2013).

\section{Determinación de Grasa}

Se colocaron las muestras en estufa a $110^{\circ} \mathrm{C}$ por 2 horas, luego se dejaron reposar en el desecador por 20 a 30 minutos, se procedió a pesar 1 gramo de muestra y colocarlo en el extractor de grasa Goldfish, aplicando la metodología que indica la Norma INEN 
174 (Instituto Ecuatoriano de Normalización INEN, 1989).

\subsection{Análisis Organoléptico}

La valoración sensorial se realizó catadores entrenados estableciendo las principales características del cacao, entre los cuales de determinó perfil de sabores específicos: frutal, floral, nuez, dulce; perfil de sabores específicos: amargor, acidez, astringencia, sabores adquiridos: verde, moho, otros (Vera et al., 2014).

\section{RESULTADOS Y DISCUSIÓN}

\section{Humedad en el licor de cacao}

En la Tabla I se observa el análisis químico del licor de cacao encontrándose diferencia significativa $(\mathrm{p} \leq 0,05)$ entre la media de humedad para todos los tipos de cacao y las diferentes maderas, la húmeda es superior en Trinitario cuando se fermenta con la madera pino; esta diferencia puede ser debido al tipo de cacao y las condiciones de la madera, estos estudios son referenciado por la norma NTE-INEN - 0623 (Instituto Ecuatoriano de Normalización INEN, 1988), sobre la calidad del licor de cacao y mencionados por Sánchez et al., (2017) al realizar estudios de polvillos de cacao quien encontró que la humedad es superior en estos tipos de productos.

Tabla I. Características químicas del licor de cacao.

\begin{tabular}{lccccc}
\hline Nivel & Humedad & Ceniza & Grasa & pH & Acidez \\
\hline $\begin{array}{l}\text { Variedad de Cacao/ Tipo de Made- } \\
\text { ra }\end{array}$ & $2,07 \mathrm{c}$ & $2,97 \mathrm{~b}$ & $49,31 \mathrm{a}$ & $5,62 \mathrm{~b}$ & $1,17 \mathrm{~b}$ \\
T1 Nacional/Laurel & $2,20 \mathrm{~b}$ & $2,98 \mathrm{~b}$ & $50,00 \mathrm{a}$ & $5,68 \mathrm{a}$ & $1,21 \mathrm{~b}$ \\
T2 Trinitario/Guayacán Blanco & $2,26 \mathrm{~b}$ & $3,18 \mathrm{a}$ & $49,27 \mathrm{a}$ & $5,75 \mathrm{ab}$ & $1,21 \mathrm{~b}$ \\
T3 Nacional/Pino & $2,32 \mathrm{~b}$ & $3,33 \mathrm{a}$ & $51,11 \mathrm{a}$ & $5,79 \mathrm{a}$ & $1,27 \mathrm{a}$ \\
T4 Trinitario/Laurel & $2,39 \mathrm{a}$ & $3,37 \mathrm{a}$ & $50,43 \mathrm{~b}$ & $5,80 \mathrm{a}$ & $1,34 \mathrm{a}$ \\
T5 Nacional/Guayacán Blanco & $2,44 \mathrm{a}$ & $3,31 \mathrm{a}$ & $50,45 \mathrm{~b}$ & $5,82 \mathrm{a}$ & $1,32 \mathrm{a}$ \\
T6 Trinitario/Pino & 2,28 & 3,19 & 50,09 & 5,75 & 1,25 \\
Promedio & 2,44 & 3,37 & 51,11 & 5,82 & 1,34 \\
Valor máximo & 2,07 & 2,97 & 49,27 & 5,62 & 1,17 \\
Valor mínimo & 4,32 & 3,83 & 1,05 & 0,99 & 3,77 \\
Coeficiente de Variación (\%) &
\end{tabular}

Medias con una letra común no son significativamente diferentes según Tukey $(p>0,05)$. 


\section{Ceniza en el licor de cacao}

En la Tabla I se observa sobre el análisis químico del licor de cacao, no encontrándose diferencia significativa $(\mathrm{p} \leq 0,05)$ entre la media de ceniza para todos los tipos de cacao y las diferentes maderas, pero se observa diferencias estadísticas en las interacciones de la ceniza siendo superior cuando se fermenta el cacao nacional con la madera guayacán blanco, estos estudios son referenciado por Alegría, (2015) al realizar estudios del proceso de tostado de semillas de cacao quien encontró que la ceniza es superior en estos tipos de proceso.

\section{Porcentaje de grasa en el licor de cacao}

El análisis químico del licor de cacao se encontró diferencia significativa $(\mathrm{p} \leq 0,05)$ entre la media de grasa para todos los tipos de cacao y las interacciones, la grasa se manifiesta superior con el CCN 51 y las interacciones de $\mathrm{CCN} 51$ fermentada con la madera laurel, estos estudios son referenciado por Vera et al., (2014) al realizar estudios de Atributos físicos-químicos $\mathrm{y}$ sensoriales de las almendras de quince clones de cacao nacional (Theobroma cacao L.) en el Ecuador encontrando porcentajes de grasa inferiores a los reportado en este estudio.

\section{Potencial Hidrógeno $(\mathrm{pH})$ en el licor de cacao}

En la Tabla I se observa diferencia significativa $(\mathrm{p} \leq 0,05)$ entre la media de $\mathrm{pH}$ para todos los tipos de madera y las interacciones, el $\mathrm{pH}$ se manifiesta superior cuando se fermenta con madera pino y las interacciones de CCN 51 fermentada con la madera pino, estos estudios son referenciado por Vera et al., (2014) al realizar estudios de atributos fisicoquímicos y sensoriales de las almendras de quince clones de cacao Nacional (Theobroma cacao L.) en Ecuador, encontrando los valores de $\mathrm{pH}$ similares a los reportado en este estudio.

Ruíz et al., (2014), en los estudios realizados en la Influencia de la época de cosecha en la calidad del licor de cacao tipo nacional obtuvo valores similares a 5,05 y 5,86 y señala que a medida que avanzan los días de fermentación tiende a disminuir el $\mathrm{pH}$ y estabilizarse en un valor cercano a cinco, contrariamente la acidez aumenta a medida que avanza el proceso fermentativo.

\section{Acidez en el licor de cacao}

No se evidencia diferencia significativa $(\mathrm{p} \leq$ $0,05)$ entre la media de acidez para todos los tipos de cacao y madera; solo hay diferencias en las interacciones, la acidez se manifiesta superior en las interacciones de cacao Nacional fermentada con la madera Guayacán Blanco, estos estudios son referenciado por Del Aguila, (2017) al realizar estudios de determinación de cadmio y plomo en granos de cacao, frescos, secos y en licor de cacao encontrando los valores de acidez similares a los reportado en este estudio.

Según Zambrano-Jaramillo, (2018), estudios realizados en características de la acidez como parámetro químico de calidad en muestras de cacao fino y ordinario de producción nacional durante la fermentación, quien en su trabajo similar encontró acidez titulable entre 1,2 y $1,6 \%$; recalca que para obtener una acidez óptima en la almendra es muy importante un buen manejo de la fermentación, ya que durante este proceso los ácidos acético y 
láctico producidos en la pulpa son difundidos hacia el cotiledón, aumentando la acidez de la fracción interna de la almendra.

\section{Efecto de los fermentadores en las almendras de cacao}

En la tabla II y en el gráfico 1, se observa el efecto de los fermentadores sobre los granos de cacao, encontrándose diferencia significativa $(p \leq 0,05)$ entre la media de la temperatura del fermentador para todos los tipos de cacao, horas de fermentación y las respectivas interacciones, es superior la variedad cacao Nacional en el factor tiempo a las 24 horas y las interacciones con la madera Guayacán Blanco cacao Nacional, estos estudios son referenciado por Erazo-Gavilánez, (2019), al realizar estudios de "diseño de un fermentador y secador solar piloto, para dos variedades de cacao, en el Cantón El Empalme provincia del Guayas" el cual fluctúa en $23{ }^{\circ} \mathrm{C}$ y $48{ }^{\circ} \mathrm{C}$, encontrándose en el presente estudio valores similares (Guachamín \& Flores, 2007).

Tabla II. Efecto de la madera sobre la temperatura de las almendras en los fermentadores

\begin{tabular}{lllll}
\hline Nivel & Media & Error Est. & L Inferior & L Superior \\
\hline 120 & $35,68 \mathrm{c}$ & 1,219 & 33,14 & 38,23 \\
96 & $38,82 \mathrm{bc}$ & 1,219 & 36,27 & 41,36 \\
72 & $39,65 \mathrm{bc}$ & 1,219 & 37,11 & 42,19 \\
48 & $41,50 \mathrm{ab}$ & 1,219 & 38,96 & 44,04 \\
24 & $42,70 \mathrm{a}$ & 1,219 & 40,16 & 45,24 \\
Tipo madera & & & & 40,56 \\
Guayacán blanco & $38,80 \mathrm{a}$ & 0,8517 & 37,038 & 41,64 \\
Laurel & $39,88 \mathrm{a}$ & 0,8517 & 38,118 & 42,09 \\
Pino & $40,33 \mathrm{a}$ & 0,8517 & 38,568 & 42,87 \\
Variedad de cacao/ tipo de madera & & & & 38,15 \\
T1 Nacional/ Laurel & $40,38 \mathrm{~b}$ & 1,2 & 37,88 & 42,87 \\
T2 CCN-51/Guayacán Blanco & $35,66 \mathrm{a}$ & 1,2 & 33,16 & 41,87 \\
T3 Nacional/Pino & $40,38 \mathrm{~b}$ & 1,2 & 37,88 & 44,43 \\
T4 CCN-51/Laurel & $39,38 \mathrm{~b}$ & 1,2 & 36,88 & 42,77 \\
T5 Nacional/Guayacán Blanco & $41,94 \mathrm{~b}$ & 1,2 & 39,44 & 37,78 \\
T6 CCN-51/Pino & $40,28 \mathrm{~b}$ & 1,2 & & \\
\hline Promedio & $\mathbf{3 9 , 6 7}$ & & & \\
Valor máximo & $\mathbf{4 1 , 9 4}$ & $\mathbf{3 5 , 6 6}$ & & \\
Valor mínimo & $\mathbf{4 , 7 2}$ & & & \\
Coeficiente de Variación (\%) & & & \\
\hline Men & & & \\
\hline
\end{tabular}

Medias con una letra común no son significativamente diferentes según Tukey $(\mathrm{p}>0,05)$.

Análisis organoléptico del licor de cacao.

En la tabla III se observa el análisis organoléptico del licor de cacao no encontrándose diferencia significativa $(\mathrm{p} \leq$ $0,05)$ entre la media de cacao para todos los tipos de cacao y madera, la variable cacao se manifiesta superior en las interacciones de cacao Nacional fermentada con la madera Laurel, estos estudios son referenciado por R. Álvarez et al., (2018) al realizar estudios de evaluación de las propiedades sensoriales 
del licor de cacao obtenido en forma artesanal e industrial encontrando los valores de cacao similares a los reportado en este estudio. Para el atributo de perfil Floral no se encontró diferencia significativa $(\mathrm{p} \leq 0,05)$ entre la media para todos los tipos de cacao y madera, la variable cacao se manifiesta superior en las interacciones de cacao Trinitario fermentado con la madera Pino, estos estudios son referenciado por Sánchez et al., (2007) al realizar estudios de Caracterización organoléptica del cacao, para la selección de árboles con perfiles de sabor de interés comercial encontrando los valores de floral similares a los reportado en este estudio.

En referencia al atributo Nuez y Dulce no existe diferencia significativa $(\mathrm{p} \leq$ $0,05)$ entre las medias, para todos los tipos de cacao y madera, la variable cacao se manifiesta superior en las interacciones de cacao Trinitario fermentado con la madera Pino y Guayacán Blanco, estos estudios son referenciado por Moreno-Martínez et al., 2019) al realizar estudios de evaluación de las características físicas y sensoriales de licor de cacao asociadas a modelos de siembra encontrando los valores de nuez similares a los reportado en este estudio, mientras que Saavedra en la investigación colecta y estudio de las características morfológicas y organolépticas en fruta fresca y licor de árboles de cacao con atributos de poseer características de fino y de aroma, encontrando los valores de dulce similares a los reportado en este estudio (Saavedra et al., 2017).

Según Amores et al., (2006), sostienen que la fermentación insuficiente o ausencia de fermentación, influyen negativamente sobre la calidad sensorial del cacao, por lo tanto las épocas de cosecha y los porcentajes de fermentación favorecen la aparición de los aromas específicos en la mayoría de los licores. En referencia a perfil sensorial de sabores específicos no se encuentra diferencia significativa en los atributos de amargor, acidez y astringencia, la variable cacao se manifiesta superior en las interacciones de cacao Nacional fermentada con la madera Laurel y Pino, estos estudios son referenciado por Camacho, (2014) al realizar estudio de la influencia del porcentaje del clon $\mathrm{CCN}$ 51 en las características físico-químicas y organolépticas del licor de cacao procedente de Pucacaca y Hujingoyacu", encontrando los valores de amargor similares a los reportado en este estudio. En sabores adquiridos tanto para la variable verde como Moho no existe diferencia significativa $(\mathrm{p} \leq 0,05)$ coincidiendo por lo expresado por Bajaña, (2015) y Ramos, (2004) al realizar estudio de la Evaluación de la calidad de las almendras de cacao trinitario $\mathrm{y}$ de ascendencia nacional manifiesta que el sabor a moho se tributa a la humedad de la almendra durante su almacenamiento. 
Tabla III. Atributos sensoriales del licor de cacao

\begin{tabular}{|c|c|c|c|c|c|c|c|c|c|c|c|}
\hline & \multicolumn{5}{|c|}{ Perfil de Sabores específicos } & \multicolumn{3}{|c|}{ Perfil de sabores básicos } & \multicolumn{3}{|c|}{ Sabores adquiridos } \\
\hline & Cacao & Floral & Frutal & Nuez & Dulce & Amargor & Acidez & Astringencia & Verde & Moho & Otros \\
\hline $\mathrm{T} 1(\mathrm{Nac} / \mathrm{L})$ & $6,20 \mathrm{~A}$ & $5,20 \mathrm{~A}$ & $4,00 \mathrm{~A}$ & $2,20 \mathrm{~A}$ & $2,60 \mathrm{~A}$ & $5,60 \mathrm{~A}$ & $1,60 \mathrm{~A}$ & $3,20 \mathrm{~A}$ & $0,20 \mathrm{~A}$ & $\begin{array}{c}0,20 \\
\mathrm{~A}\end{array}$ & 0 \\
\hline $\begin{array}{l}\text { T2(Tri- } \\
\text { ni-Gb) }\end{array}$ & $6,00 \mathrm{~A}$ & $4,60 \mathrm{~A}$ & $3,20 \mathrm{~A}$ & $2,00 \mathrm{~A}$ & $5,40 \mathrm{~A}$ & $4,00 \mathrm{~A}$ & $2,20 \mathrm{~A}$ & $2,80 \mathrm{~A}$ & $0,20 \mathrm{~A}$ & $\begin{array}{c}0,00 \\
\mathrm{~A}\end{array}$ & 0 \\
\hline $\mathrm{T} 3(\mathrm{Nac} / \mathrm{P})$ & $4,00 \mathrm{~A}$ & $2,00 \mathrm{~A}$ & $2,20 \mathrm{~A}$ & $2,20 \mathrm{~A}$ & $2,80 \mathrm{~A}$ & $5,60 \mathrm{~A}$ & $3,20 \mathrm{~A}$ & $4,40 \mathrm{~A}$ & $0,40 \mathrm{~A}$ & $\begin{array}{c}0,60 \\
\mathrm{~A}\end{array}$ & 0 \\
\hline $\begin{array}{c}\text { T4(Tri- } \\
\text { ni/L) }\end{array}$ & $3,80 \mathrm{~A}$ & $5,40 \mathrm{~A}$ & $2,40 \mathrm{~A}$ & $1,60 \mathrm{~A}$ & $2,60 \mathrm{~A}$ & $4,80 \mathrm{~A}$ & $2,80 \mathrm{~A}$ & $3,60 \mathrm{~A}$ & $0,40 \mathrm{~A}$ & $\begin{array}{c}0,40 \\
\mathrm{~A}\end{array}$ & 0 \\
\hline $\begin{array}{c}\mathrm{T} 5(\mathrm{Nac} / \\
\mathrm{Gb})\end{array}$ & $4,80 \mathrm{~A}$ & $4,40 \mathrm{~A}$ & $1,00 \mathrm{~A}$ & $2,40 \mathrm{~A}$ & $2,00 \mathrm{~A}$ & $4,20 \mathrm{~A}$ & $2,80 \mathrm{~A}$ & $4,60 \mathrm{~A}$ & $0,20 \mathrm{~A}$ & $\begin{array}{c}0,20 \\
\mathrm{~A}\end{array}$ & 0 \\
\hline $\begin{array}{c}\text { T6(Tri- } \\
\text { ni/P) }\end{array}$ & $5,60 \mathrm{~A}$ & $5,60 \mathrm{~A}$ & $1,60 \mathrm{~A}$ & $2,60 \mathrm{~A}$ & $2,20 \mathrm{~A}$ & $5,00 \mathrm{~A}$ & $3,80 \mathrm{~A}$ & $4,20 \mathrm{~A}$ & $1,40 \mathrm{~A}$ & $\begin{array}{c}0,00 \\
\mathrm{~A}\end{array}$ & 0 \\
\hline Media & 5,06 & 4,53 & 2,4 & 2,16 & 2,93 & 4,86 & 2,73 & 3,8 & 0,46 & 0,23 & 0 \\
\hline CV (\%) & 13,89 & 2,86 & 32,81 & 11,98 & 30,95 & 11,29 & 21,9 & 14,02 & 77,97 & 73,29 & 0 \\
\hline
\end{tabular}

Medias con una letra común no son significativamente diferentes según Tukey $(\mathrm{p}>0,05)$.

$\mathrm{Nac} / \mathrm{L}=$ Cacao Nacional, fermentador de laurel.

Trini-Gb = Cacao Trinitario, fermentador de Guayacán blanco

$\mathrm{Nac} / \mathrm{P}=$ Cacao Nacional, fermentador de Pino

Trini-L = Cacao Trinitario, fermentador de Laurel

$\mathrm{Nac} / \mathrm{Gb}=$ Cacao Nacional, fermentador de Guayacán blanco

Trini- $\mathrm{P}=$ Cacao Trinitario, fermentador de Pino

C.V.= Coeficiente de Variación.

\section{Determinación del porcentaje de fermentación}

La investigación permitió lograr identificar el porcentaje máximo y mínimo de almendras bienfermentadas, medianamente fermentadas, violetas, pizarrosos. A grandes rasgos se deduce que los tres tipos de maderas (Pino, Guayacán Blanco, Laurel) son adecuadas para la buena fermentación de la almendra, dentro de los granos violetas se observó que si cumplieron con lo que establece la norma que es el máximo de 21 almendras, sin embargo, se detecta que en los granos pizarrosos supera el rango establecido, que es de 12 máximo, este inconveniente se localiza en mayor proporción en las maderas de Guayacán
Blanco y Pino en las dos variedades de cacao, siendo el cacao Nacional en Laurel el único que se mantiene dentro de los parámetros establecidos. 
Figura I. Descripción del porcentaje de fermentación obtenido en las dos variedades en estudio

Gráfico de Interacciones

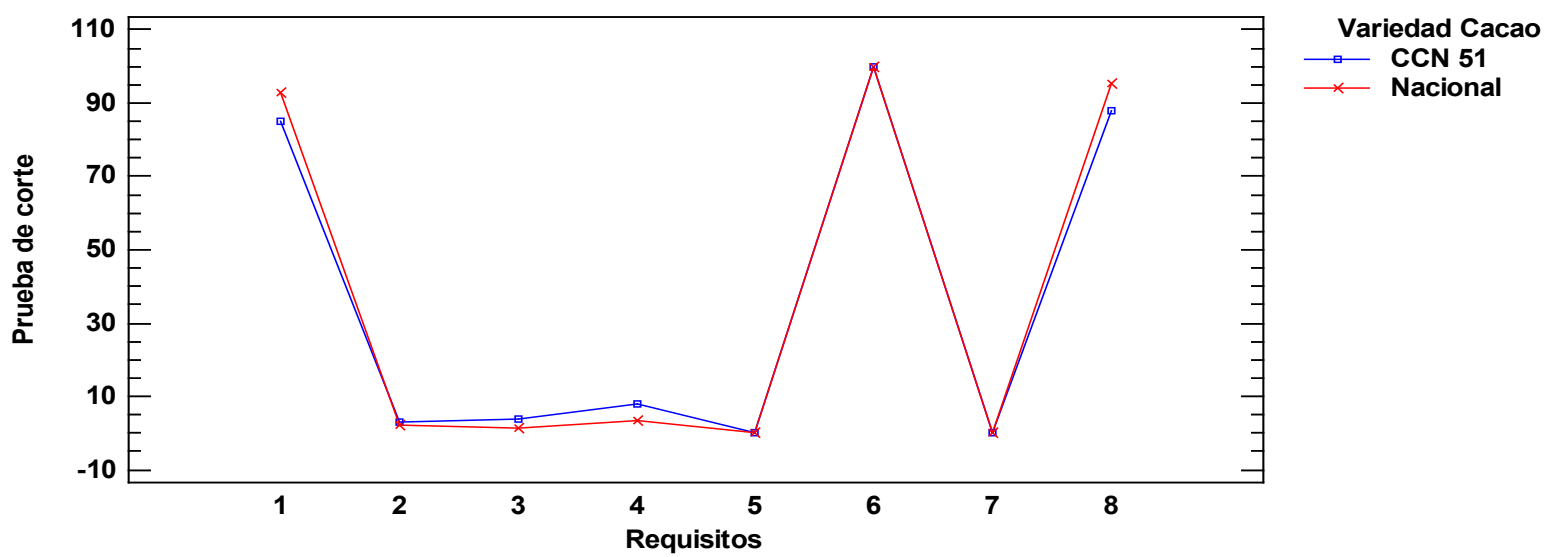

Según Wakao, citado por Erazo ((Erazo et al., 2019)), la composición química de los granos de cacao fermentado (Tabla I), depende de varios factores entre los que se puede citar: Tipo de cacao, grado de madurez, calidad de la fermentación y el secado y además el subsiguiente procesamiento de los granos (tostado).

Según Ortiz Ortiz et al., (2009), en su estudio realizado detalla que los índices físicos de calidad de los granos secos se relacionan con el grado de madurez de las almendras, es decir, mazorcas que no están completamente maduras dan origen a granos insuficientemente fermentados, violetas $\mathrm{y}$ pizarrosos. De acuerdo con Álvarez et al., (2010), en su investigación observaron un buen grado de fermentación según la prueba de corte de calidad, obteniéndose más del 80 $\%$ de granos fermentados y secos en diferentes intervalos en la remoción de la masa (cada 24 horas durante los cinco días que duró la fermentación).

Para próximas investigaciones se recomienda analizar los compuestos naturales de la madera utilizada en la fermentación de cacao, antes durante y después del uso de los cajones, lo que permitirá cuantificar químicamente la transferencia de sustancias que mejoren la calidad del cacao.

\section{CONCLUSIONES}

Mediante la fermentación de las almendras se obtuvo la temperatura máxima de 44,52 ${ }^{\circ} \mathrm{C}$ en el tercer micro fermentador con la variedad del cacao Nacional en la madera Pino y Laurel, mientras que la temperatura mínima fue de $35,66{ }^{\circ} \mathrm{C}$ con el primer micro fermentador en la variedad CCN-51 con la madera Guayacán Blanco.

En el aspecto del análisis sensorial del licor de cacao en el perfil de sabores específicos en cacao el T1 (Nacional-Laurel), Floral el T6 (CCN-51-Pino), Dulce el T2 (CCN-51 GB) obtuvieron mejores atributos, en el perfil de sabores básicos el T1 Y T3 (Nacional-Pino) presento promedio alto en su amargor, T6 tiene una acidez media en el licor de cacao, T5 (Nacional-Guayacán Blanco) obtiene una media en astringencia y sabores adquiridos Verde y Moho el T6 y T3 presentaron 
promedios altos en el licor de cacao.

Dentro de los análisis bromatológicos el licor de cacao presentó un porcentaje de humedad superior en CCN-51- Pino, mientras en ceniza tuvo un promedio alto en cacao Nacional con la madera Guayacán Blanco, en grasa presenta un alto porcentaje el cacao CCN-51 en Laurel; el pH del licor de cacao es alto con CCN-51 en Pino, el licor de cacao presenta un porcentaje alto en acidez en la variedad Nacional con la madera Guayacán Blanco.

\section{REFERENCIAS BIBLIOGRÁFICAS}

Alegría, E. (2015). Evaluación de tratamientos previos al proceso de tostado de semillas de cacao para el diseño del área de producción de pasta de cacao (Theobroma cacao). Universidad Técnica Nacional.

Álvarez, C., Tovar, L., García, Héctor, Morillo, Franklin, Sanchez, P., Girón, C., \& De Farias, A. (2010). Evaluación de la calidad comercial del grano de cacao (Theobroma cacao L.) usando dos tipos de fermentadores. Revista Cientifica UDO Agrícola, 10(1), 76-87.

Álvarez, R., Portillo, E., Portillo, A., \& Villasmil, R. (2018). Evaluación de las propiedades sensoriales del licor de cacao (Theobroma cacao L.) obtenido en forma artesanal e industrial. Revista Cien. Tecn. Agrollanía, 15, 1-6.

Amores, F., Jiménez, J., \& Peña, G. (2006). Influencia del tiempo de fermentación y el tostado sobre el desarrollo de compuestos aromáticos asociados al sabor a chocolate en almendras de caco de la variedad Nacional. 15th International Cocoa Research Conference San, 1-5.

Anabel Sánchez Campuzano, V., Agr Msc Alfonso Vasco Medina Quevedo, I., \& Ríos, L. (2007). Caracterización organoléptica del cacao (Theobroma cacao L.), para la selección de árboles con perfiles de sabor de interés comercial. [Universidad Técnica Estatal de Quevedo]. http://infocafes.com/ portal/wp-content/uploads/2017/03/ Caracterizacion_organoleptica_cacao-_ Theobroma-cacao-L._seleccion arboles_-perfiles_sabor_interes_ comercial.pdf

Armijos, A. (2002). Características de la acidez como parámetro químico de calidad en muestras de cacao (Theobroma cacao L.) fino y ordinario de producción nacional durante la fermentación. Pontificia Universidad Católica del Ecuador.

Association of Analytical Communities. (2020). AOAC 970.21-1974, PH de productos de cacao. Método potenciométrico - \$14.15: Método oficial de AOAC. 2020. http:// www.aoacofficialmethod.org/ index.php? main_page $=$ product info\&products_id $=1707$

Bajaña, C. (2015). Evaluación de la calidad de las almendras de cacao trinitario y de ascendencia nacional en la zona de Vinces. Universidad de Guayaquil.

Camacho, C. (2014). Influencia del porcentaje 
del clon CCN 51 en las características fisicoquímicas y organolépticas del licor de cacao procedente de Pucacaca y Huingoyacu. Universidad Nacional Agraria de la Selva.

Chang, J. V.(2018). Guíapara elmejoramiento de cacao nacional. June. https://doi. org/10.13140/RG.2.2.12413.26089

Del Aguila, E. (2017). Determinación de cadmio y plomo en granos de cacao, frescos, secos y en licor de cacao (Theobroma cacao) [Universidad Agraria Nacional de la Selva]. In Universidad Nacional Agraria de la Selva. http://repositorio.unas.edu.pe/ handle/UNAS/1269

Erazo, C., Gallegos, W., Coral, K., \& Arisqueta, L. (2019). Diseño de un fermentador y secador solar piloto, para dos variedades de cacao (Theobroma cacao L) en el cantón el Empalme provincia del Gu ayas. Universidad Nacional Sek.

Giraldo Palencia. (2016). La fermentación del cacao en madera mejora su sabor. Septiembre.

Guachamín, C., \& Flores, P. (2007). Diseño de un secador de cacao Fino [Universidad Politécnica Nacional]. http://bibdigital. epn.edu.ec/bitstream/15000/790/1/CD1238.pdf

Instituto Ecuatoriano de Normalización. (2013). NTE INEN 533:2013 Cacao (Productos derivados). Determinación de ceniza total.

Instituto Ecuatoriano de Normalizacion
INEN. (2013). Norma Técnica Ecuatoriana NTE INEN 1676: 2013 Primera revisión Productos derivados de cacao. Determinación de la humedad o pérdida por calentamiento. Método gravimétrico.

Instituto Ecuatoriano de Normalización INEN. (1988). NTE INEN 0623 Pasta (Masa, licor) de cacao. Requisitos.pdf.

Instituto Ecuatoriano de Normalización INEN. (1989). NTE INEN 174. Cacao en grano. Determinación del contenido de grasa.

Instituto Ecuatoriano de Normalización INEN. (2006). NTE INEN 0176: Cacao en grano. Requisitos (Vol. 0176). http://www.agrocalidad.gob.ec/ wp-content/uploads/2016/03/NTE.INEN-0176.2006.pdf

Morales, W., Vallejo, C., Sinche, P., Torres, Y., Vera, J., \& Anzules, E. (2016). Mejoramiento de las características físico-químicas y sensoriales del cacao CCN51 a través de la adición de una enzima y levadura durante el proceso de fermentación. Revista Amazónica Ciencia y Tecnología, 5(2), 169-181.

Moreno-Martínez, E., Gavanzo-Cárdenas, Ó. M., \& Rangel-Silva, F. A. (2019). Evaluación de las características físicas y sensoriales de licor de cacao asociadas a modelos de siembra. Ciencia y Agricultura, 16(3), 75-90. https://doi.org/10.19053/01228420.v16. n3.2019.9890

Ordoñez, S., Vera, J., \& Tigselema, S. (2019). 
Cascarilla de cacao (Theobroma Cacao

1.) De líneas híbridas para la elaboración de rehiletes de chocolate. Universidad y Sociedad, 11(2), 136-141.

Ortiz, L., Graziani, L., \& Rovedas, G. (2009). Influencia de varios factores sobre características del grano de cacao fermentado y secado al sol. Agronomía Tropical, 59(2), 119-127.

Ramos, G. (2004). La Fermentación, el Secado y Almacenamiento del Cacao. En Taller Internacional de Calidad Integral de Caco, Teoría y Práctica.

Rivera Fernández, R. D., Barrera Álvarez, A. E., Guzmán Cedeño, Á. M., Medina Quinteros, H. N., Casanova Ferrín, L. M., Peña Galeas, M. M., \& Nivela Morante, P. E. (2012). Efecto del tipo y tiempo de fermentación en la calidad física y química del cacao (Theobroma cacao L.) Tipo Nacional. Ciencia y Tecnología, 5(1), 7-12. https://doi. org/10.18779/cyt.v5i1.165

Ruíz, M., Mera, O., Prado, Á., \& Cedeño, W. (2014). Influencia de La época de cosecha en la calidad del licor de cacao tipo Nacional. Espamciencia, 5(2), 7987. http://espamciencia.espam.edu.ec/ index.php/Revista_ESPAMCIENCIA/ article/view/85/71

Saavedra, R., Cárdenas, H., Márquez, K., Beraun, Y., Carranza, M., Hurtado, O., \& Chia, J. (2017). Colecta y estudio de las características morfológicas y organolépticas en fruta fresca y licor de arboles de caco (Theobroma cacao L.) con atributos de poseer caracteristicas de fino $y$ de aroma. International Symposium on Cocoa Research (ISCR), Lima, Peru, 13-17 November 2017 “COLECTA, 1-11. https://www. researchgate.net/publication/327872259

Sánchez, Á., Naranjo, J., Córdova, V., Ávalos, D., \& Zaldívar, J. (2017). Caracterización bromatológica de los productos derivados de cacao (Theobroma cacao L.) en la Chontalpa, Tabasco, México. Revista Mexicana de Ciencias Agrícolas, 14(14), 28172830. https://doi.org/10.29312/remexca. v0i14.453

Servicio Ecuatoriano de Normalización INEN. (2017). Norma Técnica Ecuatoriana NTE INEN 620.

Vallejo, C., Loayza, G., Morales, W., \& Vera, J. (2018). Sensory profile of genotypes of cocoa (Theobroma cacao L.) un the parish of Valle Hermoso - Ecuador. Revista ESPAMCIENCIA Para El Agro, 9(2), 103-113. https://dialnet.unirioja. es/servlet/articulo?codigo $=7020076$

Vera, J., Vallejo, C., Párraga, D., Morales, W., Macías, J., \& Ramos, R. (2014). Atributos físicos-químicos y sensoriales de las Almendras de quince clones de cacao nacional (Theobroma cacao 1.) en el Ecuador). Ciencia y Tecnología, 7(2), 21-34. 